\title{
Inguinoscrotal pathology
}

\author{
Luis Guerra, MD; Michael Leonard, MD
}

Department of Surgery, Division of Pediatric Urology, Children's Hospital of Eastern Ontario, University of Ottawa, Ottawa, ON, Canada

Cite as: Can Urol Assoc J 2017;11(1-2Suppl1):S41-6. http://dx.doi.org/10.5489/cuaj.4336

See related commentary on page $\$ 47$.

\section{Abstract}

Infants, children, and adolescents with inguinoscrotal pathology comprise a significant proportion of emergency department and outpatient visits. Visits to the emergency department primarily comprise individuals presenting with scrotal pain due to testicular torsion or torsion of the testicular appendages. At such time, immediate urological consultation is sought. Outpatient visits comprise those individuals with undescended testes, hydroceles, and varicoceles. Rare, but important problems, such as pediatric testicular tumours, may also present in the office setting. Many of these outpatient visits are to primary care physicians, who should have an appreciation of the timing and need for referral.

The purpose of this review is to familiarize the general urologist and primary care physician with these varied pathologies and give insight into their assessment and management. Some of these same conditions are seen in adult patients, but there are some significant differences in their management in the pediatric group. In addition, the utility of imaging studies, such as ultrasound, are discussed within each pathological entity. It is hoped that this overview will assist our general urology and primary care colleagues in patient management for diverse inguinoscrotal pathologies.

\section{Undescended testis}

Undescended testis has a prevalence of $3 \%$ of all term baby boys, but can be seen in $30 \%$ of premature infants. Only $1 \%$ will be undescended at one year of age. ${ }^{1}$ Testes are formed at the lumbar level of the posterior abdominal wall from the intermediate mesoderm within the urogenital ridges of the embryo. They migrate in a predefined pathway from the lumbar area towards the inguinal canal and reach the scrotum. This descent may be arrested at some point along the normal pathway, which characterizes the undescended testis. Ectopic testes are deviated to a position off this normal path. The lack of palpable testes within the scrotum can be detected during the first postnatal exam, however, due to the small genitalia in the newborn, the condition may be missed at this moment. Subsequent visits to the family physician for well-baby checkups will definitely be the best opportunity for the diagnosis of an undescended testis. Eighty percent of the undescended testes are palpable at the level of the inguinal canal or some surrounding ectopic sites, such as pre-penile, perineal, thigh, femoral canal, and the pouch of Denis Browne (the space located between the aponeurosis of the external oblique muscle and the Scarpa's fascia). ${ }^{2}$ In most cases, the testicle will descend spontaneously to the scrotum by six months to one year of age; if this does not occur, the patient should be referred to urology and then surgical treatment is recommended.

Surgery will depend on the location of the testis, and if the testis is palpable or not. A typical palpable testis is usually suitable for an open orchidopexy through an inguinal or scrotal incision, dissection to elongate the spermatic cord, closure of the patent processus vaginalis, and a creation of a scrotal subdartos pouch to pex it inside the scrotum. Generally, ultrasound is not indicated for diagnosis of undescended testes. ${ }^{3,4}$ In surgical planning, ultrasound can help to identify a non-palpable testis, especially if the testis is inguinal. If the testis is located inside the abdomen or is not seen on ultrasound, laparoscopy is recommended. The Fowler-Stephens maneuver, comprising spermatic vessel transection, can be done to help bring the testis down either at the same time (one stage) or four to six months later (two stages) after development of collateral vessels.

\section{Testicular fumours}

Testicular tumour incidence is $0.5-2$ per 100000 children and accounts for $1-2 \%$ of all pediatric solid tumours. ${ }^{5}$ The most common age at presentation is two years and again at puberty. Benign lesions represent a greater percentage of cases in children than in adults and germ cell tumours account for two-thirds of pre-pubertal testicular tumours. Previous historical series reported yolk sac (YST) as the 
most prevalent testicular tumour in children, ${ }^{6,7}$ however, more recent reports show that teratomas, which are benign tumours, are the most common histological type in prepubertal patients. ${ }^{8,9}$ It should be clearly stated however, that YST is the most common malignant testicular tumour in children. Teratomas contain the three embryologic layers (endoderm, mesoderm, and ectoderm) and may give rise to any of the tissues derived from them. Metastases seen in teratomas are usually caused by an overlooked YST component on the pathology.

The most common presentation in a child with testicular tumour is a painless scrotal mass, but mild tenderness and heaviness with dull ache may be present. In infants and toddlers, the mass is usually found when they are bathing or showering. A small hydrocele may be present. Among scrotal masses in the pediatric group, adolescents might present with longer time from onset of symptoms to diagnosis. Nogueira Neto, in a series of 60 patients (34 children and 26 adolescents) with testicular or paratesticular neoplasms, reported that the mean length of time from the onset of symptoms to the diagnosis in adolescents and children, were 4.9 and 2.3 months, respectively. ${ }^{10}$

Ultrasound is the first imaging study used for assessment of testicular masses and has high accuracy. A solid mass involving the testis may have calcification or cystic areas. YSTs are usually solid and heterogeneous on ultrasound. Teratomas tend to have complex cystic and solid areas with calcifications, while Sertoli and Leydig cell tumours appear as hypoechogenic lesions on ultrasound. ${ }^{11}$ Epidermoid cysts have a central hypoechoic area surrounded by an echogenic rim on ultrasound, which gives the typical image called "onion skin" if multiple concentric rings are present. Serum markers will include alpha-fetoprotein (AFP), beta subunit of human chorionic gonadotropin ( $\beta$-hCG), and lactate dehydrogenase (LDH). AFP has a half-life of five days and one should note that it can be normally elevated in infants. ${ }^{12}$ AFP is elevated in $90 \%$ of YSTs and in these cases, parenchymal-sparing surgery should not be done. $\beta$-hCG is rarely increased in pre-adolescent tumours and has a half-life of 24 hours. Serum markers are used for monitoring the treatment result and should be normal range after five half-lives; they can also be used to detect relapse.

Chest X-ray (or computed tomography [CT]) and abdominal CT are indicated at diagnosis to rule out lung and retroperitoneal metastases. Benign lesions represent a greater percentage of cases in children than in adults. Pohl et al reviewed four pediatric centres and reported on 98 prepubertal patients. They found teratoma in $48 \%$ of children, YSTs in 15\%, and epidermoid cyst in 14\%; other types were juvenile granulosa cell in 5\%, Leydig cell in $4 \%$, Sertoli cell in $3 \%$, and mixed gonadal stromal in $1 \% .{ }^{13} \mathrm{~A}$ pathognomonic pathology finding in YSTs are Schiller-Duval bodies, described as a small papilla with a central fibrovascular core that protrudes into a central cystic space. Around $80-90 \%$ of YSTs are Stage I, but up to $20 \%$ can have hematogenous lung metastases at presentation, leaving the retroperitoneum spared; that is the reason why retroperitoneal lymphadenectomy is not commonly done unless there is an elevated AFP or retroperitoneal metastases post-chemotherapy. ${ }^{14}$ The Children's Oncology Group staging system uses imaging, surgical and pathological information to help guide multimodal therapy (Table 1). Testicular tumours in adults are more likely to be malignant neoplasms and are managed with radical orchiectomy in this age group. Classically, the management of testicular tumour in children has followed the adult protocol, with radical inguinal orchiectomy being the recommended approach. However, the likelihood of malignancy is significantly lower in prepubertal patients and, at this age, a conservative approach with partial orchiectomy can be considered in prepubertal patients with normal AFP levels and a tumour that is technically resectabl, ${ }^{11}$ such as in small teratomas and epidermoid cysts. In children, the oncological surgical principles for radical orchiectomy are the same as in adults. As previously mentioned, retroperitoneal lymphadenectomy is not routinely done in the pediatric population unless there is evidence of post-chemotherapy lymph node metastases. Platinum-based chemotherapy is very effective for YSTs. Radiotherapy usually has no application in children.

\section{Acute scrotum}

\section{Testicular torsion}

Testicular torsion is one of the most common surgical emergencies in pediatric urology and it is caused by torsion of the spermatic cord leading to venous congestion and arterial ischemia. There are two distinct mechanism of testicular torsion: extravaginal (most common at the perinatal period) and intravaginal (most common in older children and adolescents). In extravaginal torsion, the spermatic cord and testis twist along with the tunica vaginalis, while in intravaginal torsion, the spermatic cord and testis twist inside the tunica vaginalis. In the post-neonatal period, the latter is firmly attached to the inner wall of the scrotum.

Thirty percent of boys with acute scrotum presenting to emergency have testicular torsion and the highest incidence is around puberty. ${ }^{15}$ They can present with severe testicular pain, scrotal skin hyperemia, nausea, vomiting, and a high riding testis. They may be pale with profuse sweating and have difficulty walking. The testis may vary from slightly tense to a hard mass with rubbery consistency, depending on how long the testis has been torted. It tends to be high in the scrotum and the cremasteric reflex may be abolished. The Prehn's sign is negative: "Lifting and holding the testis 


\begin{tabular}{ll}
\hline $\begin{array}{l}\text { Table 1. The Children's Oncology Group testicular tumour } \\
\text { staging system }\end{array}$ \\
\hline Stage & \multicolumn{1}{c}{ Extent of disease } \\
\hline I & $\begin{array}{l}\text { Tumour is limited to testis, completely resected by } \\
\text { high inguinal orchiectomy. No clinical, radiographic, } \\
\text { or histological evidence of disease beyond the testis. If } \\
\text { scrotal orchiectomy has been performed, all margins are } \\
\text { negative after resection of proximal cord structures to the } \\
\text { level of the internal inguinal ring. Tumour markers are } \\
\text { negative after appropriate half-life decline. }\end{array}$ \\
Microscopic residual disease is present in the scrotum \\
or high in spermatic cord ( $<5$ cm from proximal end). \\
Tumour markers remain elevated after appropriate half- \\
life interval. Tumour rupture or scrotal biopsy before \\
complete orchiectomy. \\
Retroperitoneal lymph node involvement. Lymph \\
nodes $>4$ cm by computed tomography are considered \\
metastases. Biopsy needed to document nodal \\
metastases in lymph nodes $>2$ cm and $<4$ cm. \\
Distant metastatic deposits.
\end{tabular}

does not alleviate the pain." Prenatal torsion may present in an asymptomatic baby born with an enlarged and hard testis seen on the physical exam or noted by the parent at home; it will have no arterial flow on Doppler ultrasound. A perinatal torsion is an extravaginal torsion. The child has an increased risk of contralateral torsion in the first one to two months of life and this should be discussed with the family while discussing the management plan. The treatment of a perinatal torsion is controversial and some schools recommend immediate or delayed surgical exploration and orchidopexy of the contralateral testis, while others recommend close observation only and intervention at the first sign of contralateral torsion. The parents should participate in this informed decision.

The diagnosis of torsion is best made by a careful history and physical exam, which is usually definitive. Imaging tests are usually not helpful, but can be done to rule out differential diagnoses, such as epididymitis, tense hydrocele, inguinoscrotal hernia, or torsion of testicular appendage if testicular torsion is not highly suspected. Ultrasound should not be done if there is a high clinical suspicion for testicular torsion, as it delays the surgery. Ultrasound will reveal absence of blood flow and an inhomogeneous parenchyma. Bell Clapper deformity is a transverse lie of the testis due to a high fixation in the scrotum; Caeser and Kaplan reported prevalence of $12 \%$ in an adult autopsy series of 101 testes. ${ }^{16}$ Patients with recurrent testicular torsion-detorsion should have elective bilateral scrotal orchidopexy to avoid a missed torsion and potential loss of the testis. ${ }^{17}$

The treatment of a confirmed testicular torsion is surgical. The patient is brought immediately to the operating room for midline scrotal exploration. If the testis is salvageable, a bilateral orchidopexy is done using sutures. If there is doubt about the testicular perfusion, a small incision in the tunica albuginea will help confirm the presence of bright arterial bleeding. A dead testis with signs of early necrosis of the epididymis is best managed with ipsilateral orchiectomy and pexy of the contralateral testis. Despite attempts to preserve a testis, it may progress to atrophy due to the ischemia. There is a theoretical time window of six hours from the onset of torsion to the surgery, which is believed to give the highest chance of testicular survival. Manual spermatic cord detorsion at the bedside is controversial, as it can make the torsion worse.

\section{Torsion of testicular appendage}

A quite common differential diagnosis of acute scrotum in young children is torsion of the testicular or epididymal appendages, which are embryologic remnants of the Müllerian or Wolffian ducts, respectively. Patients present with mild to moderate pain and scrotal swelling. One may see a "blue dot sign" through the skin or feel a hard nodule at the upper poles of the testis. The cremasteric reflex is usually intact and the Prehn's sign is positive. The treatment is conservative with anti-inflammatories and analgesics. Ultrasound may show a normal testis, good arterial flow, and an enlarged inflamed epididymis with hyperemia; a round avascular nodule represents the torted appendix.

\section{Epididymitis}

Epididymitis is not a common condition in children and adolescents, but is part of the differential diagnosis of an acute scrotum. A child with recurrent epididymitis should have an ultrasound to rule out ipsilateral ectopic ureter to the seminal vesicle or, less commonly, to the vas deferens. Older children with urethral valves or obstruction may develop epididymitis. Adolescents with a history of sexually transmitted disease may present with acute epididymitis and should be treated with antibiotics and anti-inflammatories.

\section{Testicular trauma}

The testicles sit outside the abdominal cavity and are protected by scrotal skin, fasciae, and muscular layers. Theoretically, they are more susceptible to external forces; however, their mobility and tissue elasticity prevent trauma to a certain extent. Blunt trauma (sports, playground injuries, falls, and straddle trauma) is mostly seen in the pediatric population, as opposed to penetrating scrotal trauma (gunshot injuries, road accidents, sharp materials) seen in adults. At presentation, the scrotum may be enlarged, firm, and painful, and have skin bruising or laceration, depending on the mechanism of trauma. Hematocele and hematoma may be present.

Ultrasound is the best imaging test for assessment of testicular trauma and may show parenchymal fracture or 
rupture of the tunica albuginea. Despite being operatordependent, ultrasound can have a specificity and sensitivity of around $75-95 \%$ for diagnosing testicular rupture; ${ }^{18,19}$ Lee et al reported that in a series of 70 trauma cases, operative and ultrasound findings matched in almost $100 \% .^{19}$

Management depends on the severity and type of trauma. Over the last two decades, we have seen a change in the management of blunt testicular trauma, with less surgical exploration and a more conservative approach. Penetrating trauma should be explored to repair injured tissues, avoid infection, correct anatomy, and preserve testicular function.

\section{Varicocele}

Varicoceles occur in $15 \%$ of adolescent males and rarely before puberty. ${ }^{20}$ They are primarily left-sided due to the left spermatic vein inserting to the left renal vein at a $90^{\circ}$ angle. Varicoceles most commonly present as incidental findings in adolescents, although some may have dull, aching testicular pain or cosmetic embarrassment as an indication for surgery. How does one decide on intervention in the asymptomatic adolescent with a varicocele? Most studies looking at semen parameters focus on late adolescence and early adulthood, and indications for surgery would be consistent with that in the adult population. In the younger adolescent, the concept of testicular volume as a surrogate marker for fertility is paramount. One may consider left testicular hypotrophy as an indication for repair, as catch-up growth has been noted after intervention. ${ }^{21}$ Left testicular hypotrophy has been defined as a differential volume of $10-20 \%$ (RTV-LTV/RTV $x 100$ ) or 2-3 $\mathrm{ml}$ smaller as compared to the right. Testicular volumes may be calculated using an orchidometer or ultrasound. Care must be exercised in early puberty, at which time differential testicular growth can be seen, and with followup, significant catch-up growth can occur spontaneously. We and others have shown that in early adolescents with varicocele and 15-20\% testicular size discrepancy, spontaneous catch-up growth may occur in $50-70 \%$ of cases, sparing many unnecessary surgeries. ${ }^{22,23}$ However, by Tanner stage 5, individuals with left varicoceles and $20 \%$ or greater left testicular asymmetry have total motile sperm counts $<20$ million (abnormal by World Health Organization [WHO] criteria) in $67 \% .{ }^{24}$ Can we identify these individuals earlier and possibly improve their outcome? Van Batavia et al use peak retrograde flow (PRF) in the left spermatic vein, as determined by Doppler ultrasound, to aid in their decision-making. They have found a combination of $20 \%$ or greater asymmetry in combination with PRF of $38 \mathrm{~cm} / \mathrm{second}$ or greater ("20/38 harbinger") to be strongly associated with persistent or progressive asymmetry, regardless of Tanner stage at assessment. ${ }^{25}$ In such patients, they advocate surgical intervention. Once a decision for intervention is made, the procedure may vary from a high-mass ligation of the spermatic cord proximal to the vas, to that of a microscopic subinguinal varicocelectomy. The pros and cons of the varied approaches are beyond the scope of this discussion (see specific article in this supplement). The paternity outcomes after adolescent varicocele repair are hard to come by, but a $75 \%$ paternity rate was reported in men who had undergone varicocelectomy at age 15-19 for abnormal semen parameters. ${ }^{26}$ However, this data must be tempered by that from Belgium, where screening for varicocele in high school is routine. Adolescents are then offered observation or antegrade sclerotherapy. Of the observation group, the paternity rate was $85 \%(61 / 72)$ vs. the sclerotherapy group at $79 \%$ $(67 / 86)$ ( $p>0.05)$. This paternity rate did not differ between the groups even when the left testis was smaller. ${ }^{27}$ Thus, the picture is less than clear, and the choice for or against surgical intervention should involve a fulsome discussion of the above issues with the young man and/or his parents. At the very least, followup with sequential testicular volume measurements to determine whether left testicular hypotrophy is persistent and/ or worsening, should be recommended. Older adolescents or young adults could also be offered semen analysis as another tool in the decision-making armamentarium.

\section{Epididymal cysts}

Epididymal cysts are benign and seen in $5-20 \%$ of children and adolescents. They are commonly detected when ultrasound is done to assess scrotal pain or mass. ${ }^{28}$ Such cysts are rarely the cause of pain and, in many cases, are incidental. There is some evidence that epididymal cysts may be part of the testicular dysgenesis syndrome, whereby endocrine disruptors can influence the development of the male genitalia in the embryo. This would explain the association of epididymal cysts with cryptorchidism and their well-known occurrence in the setting of maternal diethylstilbestrol (DES) ingestion. There is also an association with vasal obstruction, as seen in cystic fibrosis and with genetic conditions such as Von Hippel Lindau disease. ${ }^{29}$ In many cases, the term epididymal cyst is used interchangeably with spermatocele. The latter can only occur post-pubertally and can be distinguished from the former only by aspiration and detection of sperm in the aspirate. In most cases, epididymal cysts can be managed conservatively and may remain stable or spontaneously regress. ${ }^{28}$ If sizeable and causing cosmetic distress or pain, they can be surgically excised.

\section{Hydroceles}

Hydroceles in the pediatric age group are generally of three types. Communicating hydroceles comprise a patent processus vaginalis, which allows fluid to move between the peritoneal cavity and the tunica vaginalis surrounding the testis. Non-communicating hydroceles comprise fluid within the tunica vaginalis surrounding the testis, with an 
obliterated processus vaginalis proximally. Hydroceles of the cord comprise fluid along the spermatic cord between an obliterated processus vaginalis proximally and obliterated tunica vaginalis distally. Distinguishing between the types of hydrocele is important, as their management varies. Communicating hydroceles will often fluctuate in size and in the ambulatory child, may be minimal in the morning and increase in size as the day progresses. The fluid can usually be reduced with gentle pressure and the testis palpated. If significant groin swelling is present on history and/or an inguinal mass is appreciated on exam, then the patient likely has an indirect inguinal hernia, which is more the realm of the pediatric surgeon. Ultrasound is not a useful adjunct in diagnosis of communicating hydroceles/inguinal hernias. Communicating hydroceles have a tendency to resolve within the first year or two of life and, therefore, surgical management should be postponed until past that time. An inguinal approach with high ligation of the patent processus vaginalis and drainage of the fluid surrounding the testis is generally undertaken. ${ }^{30}$ Non-communicating hydroceles tend not to vary in size, but may increase in volume over time. Usually, they are tense and the underlying testis is not easily palpated. Since such hydroceles may be secondary to underlying testicular pathology (inflammation, tumour) and if the testis cannot be palpated, scrotal ultrasound is indicated to assure normalcy of the gonad. If present in infants and children, they may resolve. If not, they can be approached inguinally to ensure no communication is present. In adolescents, they usually persist or increase in size, and if they cause discomfort or cosmetic embarrassment, then a scrotal hydrocelectomy is indicated. Hydroceles of the cord are unlikely to resolve and are usually approached by inguinal exploration after one year of age. ${ }^{31}$

\section{Scrotal anomalies}

The scrotum forms by fusion of the fetal labioscrotal folds under the influence of dihydrotestosterone between 8-16 weeks of gestation. ${ }^{32}$ This explains the association of bifid scrotum with severe hypospadias, as hormonal factors are likely at play. In addition, the labioscrotal folds may fail to migrate infero-medially, resulting in penoscrotal transposition. These scrotal anomalies are usually corrected at the time of hypospadias repair. The occurrence of midline scrotal cysts is also accounted for by this fusion of embryological anlagen. Such cysts occur along the median raphe and may be surgically excised or left in situ, depending on the parental preference. Scrotal hypoplasia is most commonly the result of bilateral undescended testes and is not a primary scrotal anomaly. The occurrence of scrotal ectopy is rare and may be related to abnormal migration of the gubernaculum. The ectopic location may be suprainguinal (most common), infrainguinal, and perineal. Such anomalies are associated with cryptorchidism, inguinal hernia, and bladder exstrophy. There is a significant association with ipsilateral upper urinary tract anomalies and, thus, a renal ultrasound is indicated. ${ }^{33}$ As can be seen, scrotal anomalies, with the exception of midline cysts, are rarely isolated problems. The need for further investigations to rule out such issues as disorders of sexual differentiation or upper tract anomalies will depend on the clinical presentation.

Competing interests: The authors report no competing personal or financial interests.

This paper has been peer-reviewed.

\section{References}

1. Abaci A, Catli $G$, Anik $A$, et al. Epidemiology, classification and management of undescended testes: Does medication have value in its treatment? I Clin Res Pediatr Endocrinol 2013;5:65-72. https://doi.org/10.4274/Jcrpe.883

2. Herzog B, Steigert M, Hadziselimovic F. Is a testis located at the superficial inguinal pouch (Denis Browne pouch) comparable to a true cryptorchid testis? J Urol 1992;148:622-3.

3. Choosing Wisely Canada initiative. Oct 2014. http://www.choosingwiselycanada.org/recommendations/ urology/. Accessed Sep 2016

4. Tasian GE, Copp HL. Diagnostic performance of ultrasound in non-palpable cryptorchidism: A systematic review and meta-analysis. Pediatrics 2011;127:119-28. https://doi.org/10.1542/peds.2010-1800

5. Brosman, SA. Testicular tumours in prepubertal children. Urology 1979;13:581-8. https://doi.org/10.1016/0090-4295(79)90375-3

6. Ross JH, Rybicki L, Kay R. Clinical behaviour and a contemporary management algorithm for prepubertal testis tumours: A summary of the prepubertal testis tumour registry. J Urol 2002; 168:1675-9. https://doi.org/10.1016/S0022-5347(05)64386-8

7. Leonard MP, Jeffs RD, Leventhal B, et al. Pediatric testicular tumours: The Johns Hopkins experience. Urology 1991;37:235-6. https://doi.org/10.1016/0090-4295(91)80297-K

8. Shukla AR, Woodard C, Carr MC, et al. Experience with testis-sparing surgery for testicular teratoma. J Urol 2004;171:161-3. https://doi.org/10.1097/01.ju.0000101185.90327.b4

9. Metcalfe PD, Farivar-Mohseni $\mathrm{H}$, Farhat $\mathrm{W}$, et al. Pediatric testicular tumours: Contemporary incidence and efficacy of testicular preserving surgery. J Urol 2003;170:2412-6. https://doi.org/10.1097/01. ju.0000097383.09743.f9

10. Nogueira Neto FB, Petrilli AS, Macedo CR, et al. Testicular tumours in children and adolescents. J Pediatr (Rio J) 2012;88:87-92. https://doi.org/10.2223/JPED.2164

11. Ferrer F. Pediatric Urologic Oncology: Bladder and Testis: in Wein, A. J., In Kavoussi, L. R., Campbell, M. F., \& Walsh, P. C. (2012). Campbell-Walsh urology. Chapter 156, page 3591 .

12. Blohm ME, Vesterling-Hörner D, Calaminus G, et al. Alpha l-fetoprotein (AFP) reference values in infants up to 2 years of age. Pediatr Hematol Oncol 1998;15:135-42.

13. Pohl HG, Shukla AR, Metcalf PD, et al. Prepubertal testis tumour: Actual prevalence rate of histological types. J Urol 2004;172: 2370-2. https://doi.org/10.1097/01.ju.0000144402.13556.74

14. Ahmed HU, Arya M, Muneer A, et al. Testicular andparatesticular tumours in the prepubertal population. Lancet Oncol 2010;11:476-83. htrps://doi.org/10.1016/S1470-2045(10)70012-7

15. Liu CC, Huang SP, Chou YH, et al. Clinical presentation of acute scrotum in young males. Kaohsiung J Med Sci 2007;23:281-6. https://doi.org/10.1016/S1607-551X(09)70410-3

16. Caesar RE, Kaplan GW. Incidence of the bell-clapper deformity in an autopsy series. Urology 1994;44:1146. https://doi.org/10.1016/S0090-4295(94)80020-0

17. Eaton SH, Cendron MA, Estrada CR, et al. Intermittent testicular torsion: Diagnostic features and management outcomes. J Urol 2005;174:1532-5. https://doi.org/10.1097/01.ju.0000177726.84913.cc

18. Chetwood BA, Eddy B. Penoscrotal trauma, In: Challacombe, S. Bott (eds.), Diagnostic Techniques in Urology, page177, Springer-Verlag London 2014. https://doi.org/10.1007/978-1-4471-2766-6_20

19. Lee SH, Bak CW, Choi MH, et al. Trauma to male genital organs: A 10-year review of 156 patients, including 118 treated by surgery. BJU Int 2008;101:211-5. 
Guerra et al.

20. Pfeiffer D, Berger J, Schoop C, et al. A Doppler-based study on the prevalence of varicocele in German children and adolescents. Andrologia 2006;39:13-9. https://doi.org/10.1111/i.1439-0272.2006.00680.x

21. Yamamoto $M$, Hibi H, Katsuno $S$, et al. Effects of varicocelectomy on testis volume and semen paramenters in adolescents: A randomized, prospective study. Nagoya J Med Sci 1995; 58:127-32.

22. Preston MA, Carnat T, Flood T, et al. Conservative management of adolescent variococele: A retrospective review. Urology 2008; 72:77-80. https://doi.org/10.1016/i.urology.2008.02.005

23. Kolon TF, Clement MR, Cartwright $L$, et al. Transient asynchronous growth in adolescent males with a varicocele. J Urol 2008;180:1111-4. htrps://doi.org/10.1016/i.juro.2008.05.061

24. Diamond DA, Zurakowski D, Baver SB, et al. Relationship of varicocele grade and testicular hypotrophy to semen parameters in adolescents. J Urol 2007;178:1584-8. https://doi.org/10.1016/i. juro.2007.03.169

25. Van Batavia JP, Badalato $G$, Fast A, et al. Adolescent varicocele — is $20 / 38$ harbinger a durable predictor of testicular asymmetry? J Urol 2013;189:1897-901. https://doi.org/10.1016/i.juro.2012.11.011

26. Pajovic B, Radojevic N. Prospective followup of fertility after adolescent laparoscopic varicocelectomy. Eur Rev Med Pharmacol Sci 2013;17:1060-3.

27. Bogaert $G$, Orye C, De Win G. Pubertal screening and treatment for varicocele do not improve chance of paternity as adult. J Urol 2013;189:2298-303. https://doi.org/10.1016/i.juro.2012.12.030
28. Rifkin MD, Kurt AB, Goldberg BB. Epididymis examined by ultrasound. Correlation with pathology. Radiology 1984;151:187-90. https://doi.org/10.1148/radiology.151.1.6701313

29. Homayoon K, Suhre CD, Steinhardt GF. Epididymal cysts in children: Natural history. J Urol 2004;171:12746. https://doi.org/10.1097/01.ju.0000110322.87053.99

30. Koski ME, Makari JH, Adams MC, et al. Infant communicating hydroceles — do they need immediate repair or might some clinically resolve? J Pediatr Surg 2010;45:590-3. https://doi.org/10.1016/i. ipedsurg.2009.06.026

31. Palmer LS. Hernias and hydroceles. Pediatr Rev 2013;34:457-64. https://doi.org/10.1542/pir.3410-457

32. Ammini AC, Sabherwal U, Mukhopadhyay C, et al. Morphogenesis of the human external male genitalia. Pediatr Surg Int 1997;12: 401-6. https://doi.org/10.1007/BF01076950

33. Elder JS, Jeffs RD. Suprainguinal ectopic scrotum and associated anomalies. J Urol 1982;127:336-8.

Correspondence: Dr. Luis Guerra, Division of Urology, Children's Hospital of Eastern Ontario, Ottawa, ON, Canada; Iguerra@uottawa.ca 